\title{
Sagnac Interferometer Based Generation of Controllable Cylindrical Vector Beams
}

\author{
Cristian Acevedo, ${ }^{1}$ Angela Guzmán, ${ }^{2}$ Yezid Torres Moreno, ${ }^{1}$ and Aristide Dogariu ${ }^{2}$ \\ ${ }^{1}$ Grupo de Óptica y Tratamiento de Señales (GOTS), Escuela de Física, Universidad Industrial de Santander, Carrera 27 Calle 9 , \\ A.A. 678, Bucaramanga, Colombia \\ ${ }^{2}$ CREOL, The College of Optics and Photonics, University of Central Florida, P.O. Box 162700, 4000 Central Florida Boulevard, \\ Orlando, FL 32816-2700, USA
}

Correspondence should be addressed to Cristian Acevedo; cristian_rvd@yahoo.com

Received 29 February 2016; Accepted 29 May 2016

Academic Editor: Wojtek J. Bock

Copyright (C) 2016 Cristian Acevedo et al. This is an open access article distributed under the Creative Commons Attribution License, which permits unrestricted use, distribution, and reproduction in any medium, provided the original work is properly cited.

\begin{abstract}
We report on a novel experimental geometry to generate cylindrical vector beams in a very robust manner. Continuous control of beams' properties is obtained using an optically addressable spatial light modulator incorporated into a Sagnac interferometer. Forked computer-generated holograms allow introducing different topological charges while orthogonally polarized beams within the interferometer permit encoding the spatial distribution of polarization. We also demonstrate the generation of complex waveforms obtained by combining two orthogonal beams having both radial modulations and azimuthal dislocations.
\end{abstract}

\section{Introduction}

It is well known that cylindrical vector beams are solutions of vector wave equation [1]. These solutions have cylindrical symmetry in both amplitude and polarization [2]. The unique optical properties of such cylindrical vector beams make them attractive for applications such as optical tweezers [3$6]$, single molecular imaging $[7,8]$, and plasmon excitation [9]. Motivated by these applications, various methods for generating cylindrical vector beams have been proposed [1020]. These methods can be generally divided into active and passive approaches [2]. For active schemes, the resonator laser is modified such that the laser beam oscillates in a desired cylindrical vector mode [10-12]. In passive approaches the wavefront of a traditional laser beam (i.e., Gaussian beam) is transformed into the desired mode [1315]. A typical passive scheme uses a spatial light modulator (SLM) because it allows a flexible way to generate and modify the selected cylindrical vector beam [16-18].

In this paper we present a novel approach for generating the desired cylindrical vector beams with controlled polarization configuration by using an optically addressable SLM incorporated into a Sagnac interferometer $[19,20]$.
This scheme enables interfering light beams that can be easily adjusted and are spatially variant in both phase and polarization.

\section{Experimental Setup}

The experimental arrangement for generating cylindrical vector beams is shown in Figure 1. A linearly polarized light from an Argon laser (JDS Uniphase, Power $100 \mathrm{~mW}$ ) with wavelength $532 \mathrm{~nm}$ in the transverse electromagnetic ground state mode TEM00 is attenuated, filtered, and collimated. The attenuator is used to control the input intensity to the Sagnac interferometer. The collimated beam is directed by a first metallic mirror (M1) towards a half-wave plate (HWP), after which the beam is redirected by a second metallic mirror (M2) towards a 50/50 polarizing beam splitter (PBS) cube. There, the incoming beam is split into a horizontally polarized beam ( $p$-component) and a vertically polarized beam $(s$ component). In the half-wave plate, the linear polarization of the laser beam is adjusted to an angle of $45^{\circ}$ to ensure that the intensity ratios of the $s$ - and $p$-components are $1: 1$. In Figure 1, the polarizing beam splitter (PBS), the two metallic mirrors (M3 and M4), and the optically addressable 


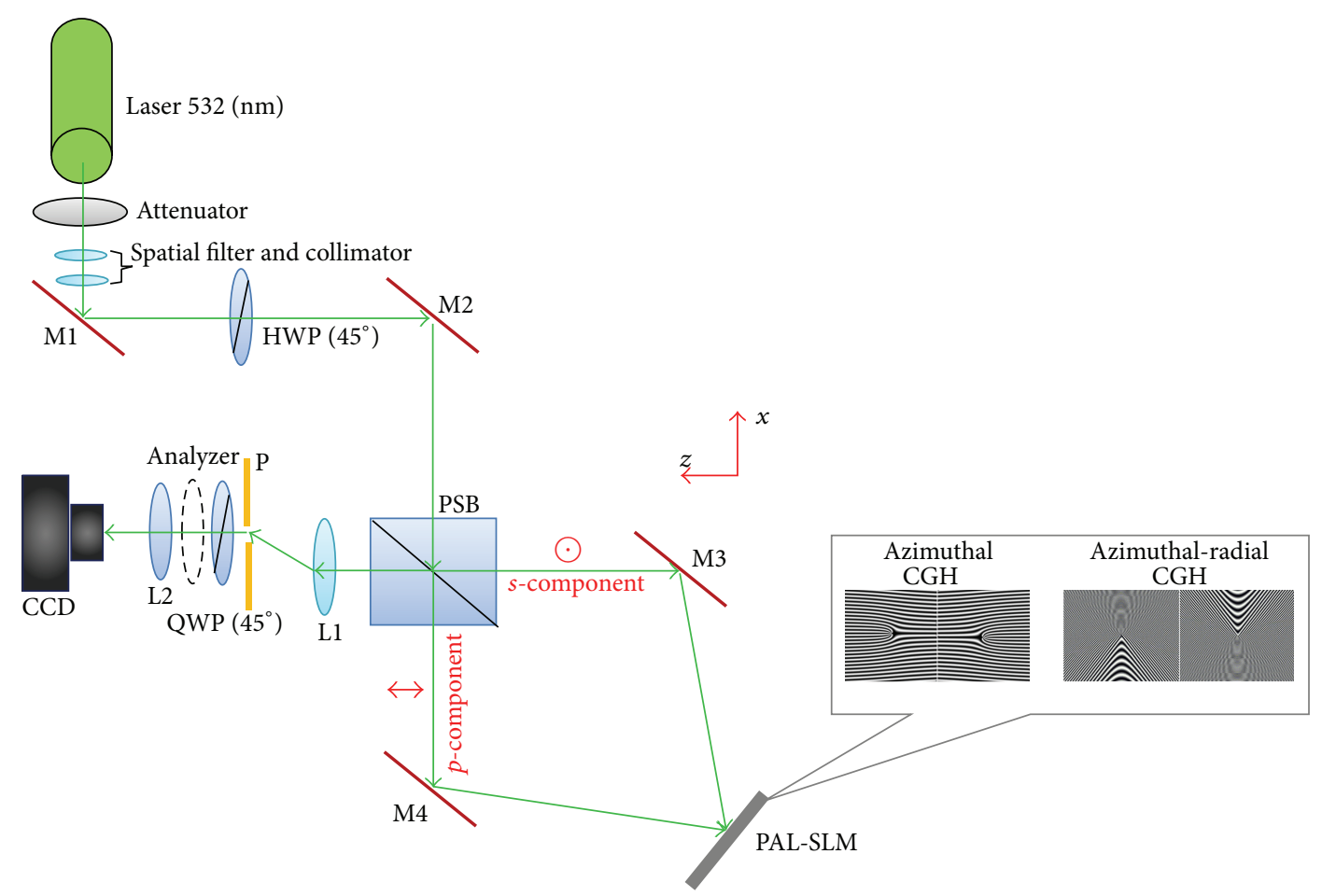

FIGURE 1: Schematic of experimental setup: attenuator; spatial filter; collimator; M, mirror; HWP, half-wave plate; PBS, polarizing beam splitter; PAL-SLM, optically addressable spatial light modulator; L, lens; P, iris diaphragm; QWP, quarter-wave plate; analyzer; and CCD. The inset shows the patterns of two compound holograms displayed on PAL-SLM for azimuthal (left) and azimuthal-radial (right) vector beams.

PAL-SLM (Hamamatsu, Model PPMX8267) compose a Sagnac interferometer. The optical paths for the $s$ - and $p$ components in the interferometer are equal; that is, $a b+$ $\mathrm{bc}+\mathrm{cd}+\mathrm{da}=\mathrm{ad}+\mathrm{dc}^{\prime}+\mathrm{c}^{\prime} \mathrm{b}+\mathrm{ba}$. Two fork computergenerated holograms (CGHs), assembled by opposite sides, are displayed on SLM window at position c (and $c^{\prime}$, resp.). The liquid crystal display LCD-coupled PAL-SLM module has a resolution of $1024 \times 768$ pixels and it is controlled in real time using Matlab software routines to compute the forked CGHs. In order to improve the diffraction efficiency of the orthogonally polarized beams, the LCD has been adjusted parallel to the polarization direction of the readout light in the phase modulation mode [21]. Each of the two components, that is, $s$ - and $p$-, is diffracted by the hologram consisting of a $2 \times 300 \times 300$-pixel window. Since the SLM is optimized only for diffraction of a horizontally polarized beam, the vertically polarized beam first passes a small half-wave plate (arranged at a $45^{\circ}$ angle) in front of the computer hologram of $s$-component that rotates its horizontal polarization to vertical state. Finally the two back-diffracted beams ( $s$ - and $p$-components) are combined at the Sagnac interferometer output.

The corresponding holograms displayed on the SLM are calculated using an amplitude reflection function given by [21]

$$
R(x, y)=\frac{1}{2} r_{0}\left[1+\cos \left(\frac{2 \pi y}{D}+\delta(x, y)\right)\right]
$$

where $r_{0}$ is the modulation depth, $D=1 / \sqrt{f_{y}^{2}}$ is the spatial period, and $\delta(x, y)$ denotes the relative phase between the orthogonal components due to the two adjacent SLM holograms. The \pm 1 orders of the $s$ - and $p$-components are recombined at the PBS and later filtered by an iris diaphragm (P) placed at the focal image plane of the Fourier lens L1 as shown in the figure. The selected orders pass through a quarter-wave plate (QWP) making an angle of $45^{\circ}$ with the horizontal axis. It transforms the two orthogonal linear polarization components of the beam, into two orthogonal circularly polarized states. Finally, the output vector beam is sent through an analyzer (rotatable linear polarizer) to a CCD camera placed close to the image plane of the Fourier lens L2. The proposed setup is very stable and highly robust against noise like vibrations or air currents, due to the fact that the two orthogonal components travel along similar paths in the Sagnac interferometer. For this reason it is possible to use this proposed interferometric method with a very short coherence length of the laser beam, for example, micrometers.

\section{Results and Discussions}

For the two adjacent CGHs placed on the PAL-SLM, the $p$ mode that is transmitted directly by the PBS at the input of the Sagnac interferometer reads $R_{p}=R$, while the $s$ mode reads $R_{s}=(1 / 2) r_{0}\left[1+\cos \left(2 \pi / D+\delta_{-}(x, y)\right)\right]$, where $\delta_{-}=\delta(-x, y)$, because the circulation of the two beams in the Sagnac interferometer is in opposite directions. The \pm 1 


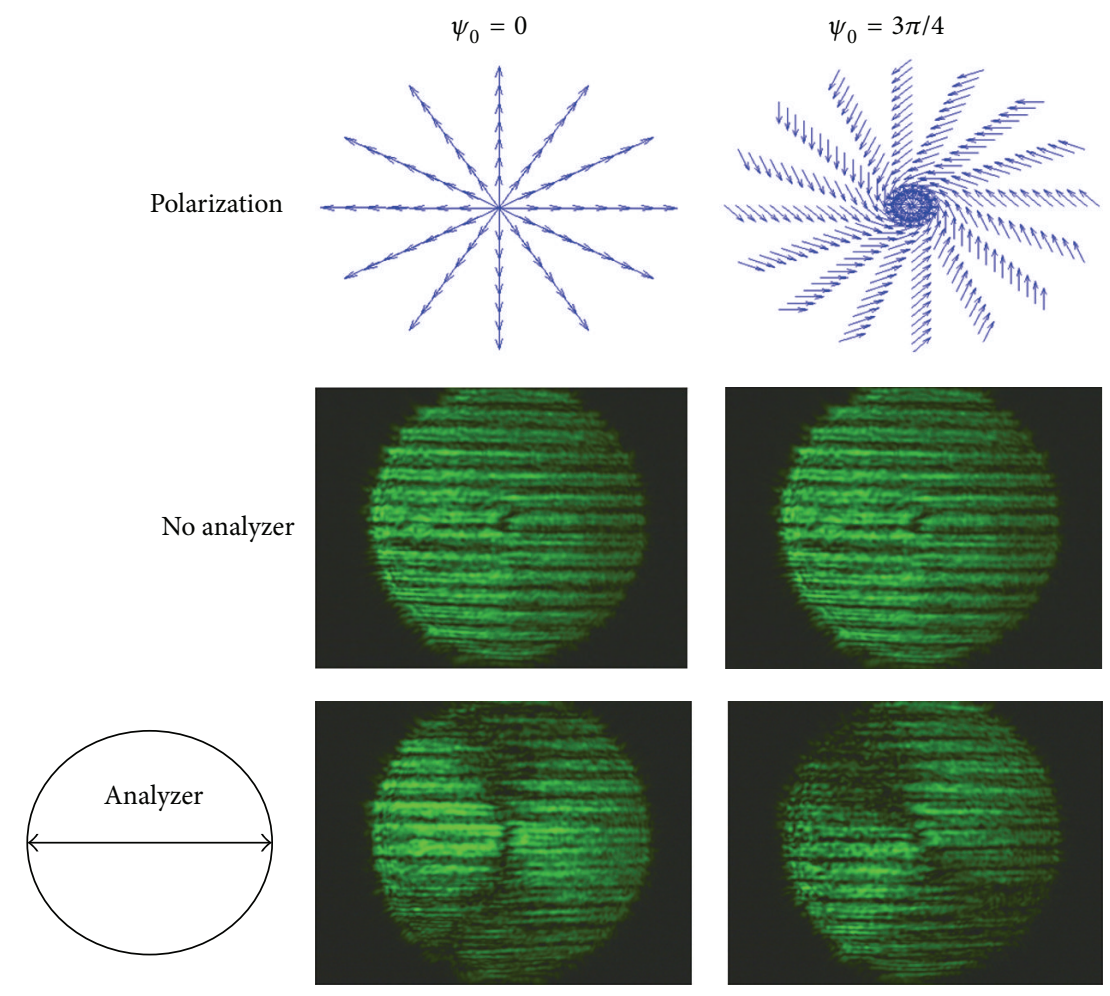

FIGURE 2: Cylindrical vector beams generated with integer topological charge $m=1$ and constant phase differences of $\psi_{0}=0$ and $\psi_{0}=3 \pi / 4$.

diffracted orders of the $p$ - and $s$-components then pass the same interferometric arrangement with phase distributions $\exp ( \pm \delta)$ and $\exp \left( \pm \delta_{-}+\psi\right)$, respectively. Here $\psi$ is a constant and controllable phase difference between components. The two beams are overlapped at the rear port of the PBS of the following form: the +1 order of $p$-component and +1 order of the $s$-component or -1 order of $p$ - and $s$-components. The iris diaphragm selects and passes through the QWP only one of the superimposed orders, for instance, the positive orders mentioned above. The resulting optical field immediately behind the QWP can be described as

$$
\begin{aligned}
& \overrightarrow{E_{s}}=\left|E_{0}\right| e^{i \delta}\left(\begin{array}{c}
1 \\
i
\end{array}\right), \\
& \overrightarrow{E_{p}}=\left|E_{0}\right| e^{i \delta_{-}+\psi}\left(\begin{array}{c}
1 \\
-i
\end{array}\right),
\end{aligned}
$$

where $E_{0}$ is a constant factor. After recombination of two optical fields into a single one at the rear focal plane of Fourier lens L2, we write the optical field as follows:

$$
\begin{aligned}
\vec{E} & =\overrightarrow{E_{s}}+\overrightarrow{E_{p}} \\
& =2 E_{0} e^{i\left(\delta-\delta_{-}+\psi\right) / 2}\left(\begin{array}{c}
\cos \left(\frac{\left(\delta-\delta_{-}+\psi\right)}{2}\right) \\
-\sin \left(\frac{\left(\delta-\delta_{-}+\psi\right)}{2}\right)
\end{array}\right) .
\end{aligned}
$$

This beam is a combination of orthogonal base vectors, left- and right-hand circularly polarized components, and describes a locally linear polarization distribution [1], where the orientation of the direction of polarization can depend on the spatial position. Equation (3) tells us that arbitrary polarization beams can be created with a well-defined phase distribution $\delta$ and by adjusting the phase difference $\psi$. The additional phase distribution $\exp \left(\left(\delta-\delta_{-}+\psi\right) / 2\right)$ is taken into account if we designed $\delta_{-}=-\delta$. We begin with the cylindrical vector beams with an integer helical phase distribution, which is represented by $\delta=m \theta$ (where $\theta$ and $m$ are the azimuth angle in the polar coordinate system and the integer topological charge, resp.). The cylindrical vector beam of the resulting field is $[\cos (m \theta-m \pi-\psi / 2), \sin (m \theta-m \pi-$ $\psi / 2)]^{T}$. This field consists of two components corresponding to left- and right-hand circular polarizations with different integer topological charge values.

First, we examine the case for $m=1$. Figure 2 illustrates the result intensity distribution of the experimental beams generated with constant phase differences of $\psi=0$ and $\psi=3 \pi / 4$, respectively. When the analyzer is not in use, the intensity distributions are the same for all values of the constant phase difference and a dark spot exists at the center of the beam due to the singularity of the optical field. When the analyzer is used, the extinction pattern appears owing to the polarization distribution in the beam cross section. Figure 3 shows a collection of cylindrical vector beams generated with integer topological charges from $m=$ 2 to $m=5$ and constant phase difference $\psi=0$. We note that the size of the central dark spot in the intensity pattern grows as $m$ increases. When an analyzer is inserted, with transmission axis to zero or ninety degrees, then the 

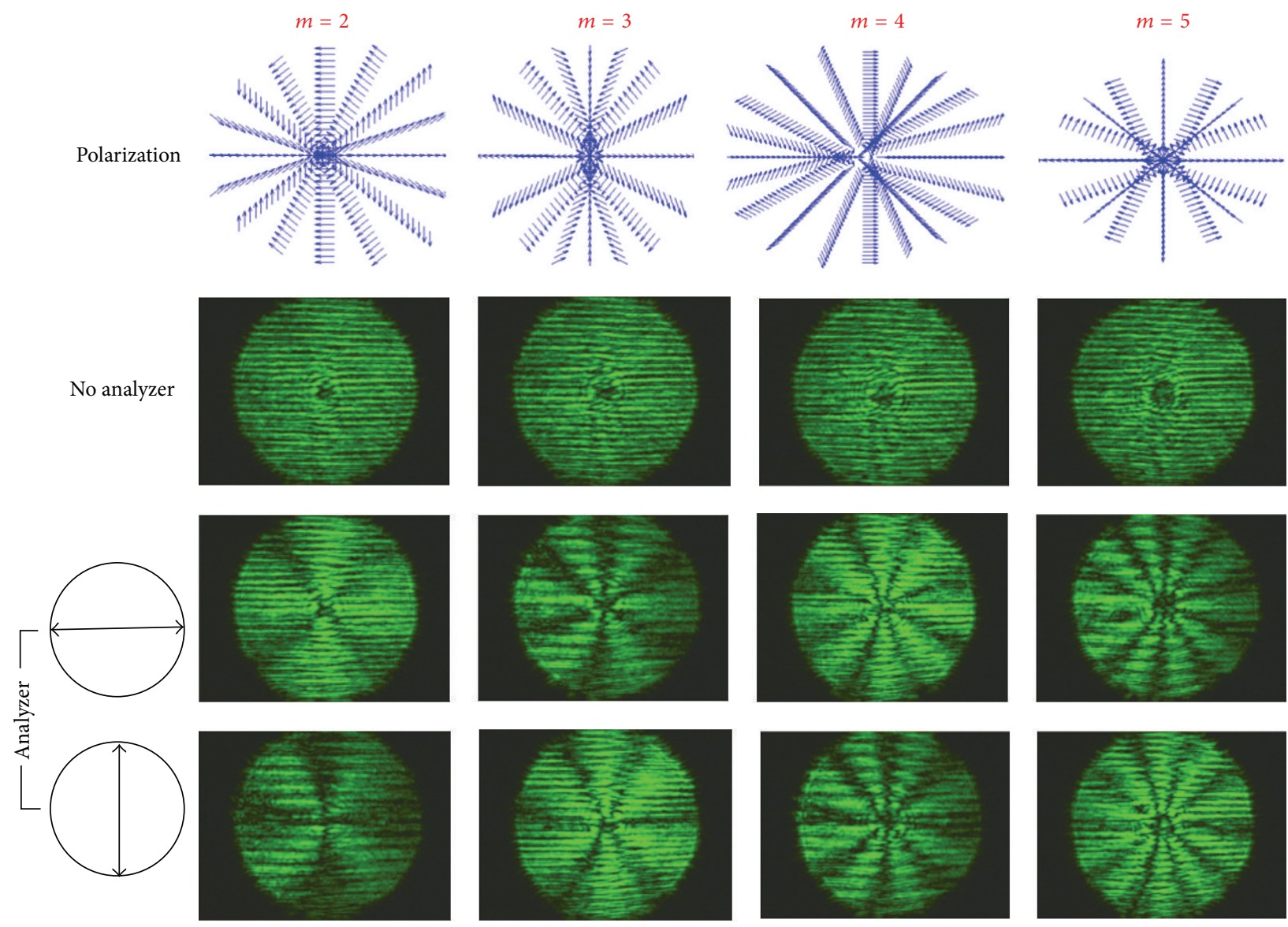

FIGURE 3: Cylindrical vector beams generated with integer topological charges from $m=1$ to 5 , all beams are generated with the same constant phase difference $\psi=0$.

extinction pattern appears again in the intensity distribution. The dark regions correspond to regions where the beam polarization is perpendicular to the analyzer. Figures 2 and 3 show that the number of both bright and dark fringes is $2 \mathrm{~m}$.

We have also investigated cylindrical vector beams with noninteger topological charge $M$ (where $M$ have integer part, $m$, and remainder, $\mu$ [22]). For that, we choose as phase distribution $\delta=M \theta$, in (3). Figure 4 shows the experimental results obtained for the values of $M=1.5$ and $M=2.5$, when $\psi=0$. The intensity pattern when there is no analyzer consists basically in the breaking of the cylindrical symmetry in the intensity distribution due to the noninteger $M$. The dark stripe, which originates by the alternating addition of helicoidal beams with the same integer topological charge $[22,23]$ and different direction of polarization, starts from the center of the intensity pattern, along the $+y$ direction. Without the analyzer, the central dark spot for $M=2.5$ is bigger than for $M=1.5$, as expected [23]. With the analyzer in place, one observes three extinction fringes for $M=1.5$ while for $M=2.5$ five different fringes appear.

The next experiment demonstrates the generation of cylindrical vector beams with azimuthal dislocation and radial modulation in the phase term of (3). In order to create this phase structure, we consider the arbitrary spatial distribution $\delta$, like the contribution of the two terms, a helical phase distribution and a radial phase distribution, related to $\theta$ and $\rho$, respectively: $\delta=n \pi \rho / \rho_{0}+m \theta$. Here $\rho$ is the radial coordinate, $\rho_{0}$ is the size of the cross beam section, and $n$ is a radial number in analogy with $m$, the azimuthal number. The CGHs for the $p$ - and $s$-components with values for $m=1$ and $n=1$ are shown in the right side of the inset in Figure 1. The corresponding experimental cylindrical vector beams for three different positive radial numbers, with values of $n=0.5$, 1.0 and $n=1.5$, are presented in Figure 5. The azimuthal number has been fixed in $m=1$. The intensity distribution of the three cylindrical vector beams without analyzer is the same. If the analyzer is inserted, the intensity distribution pattern exhibits the Archimedean Spirals where the number of the arms is independent of $n$.

Finally, if we use negative radial numbers, for example, $n=-0.5,-1.0$ and $n=-1.5$, and the analyzer is inserted, then the chirality in the intensity distribution pattern of the Archimedean Spirals changes to left-handed screw with the same number of arms as shown in Figure 6.

\section{Conclusion}

We have demonstrated an original method for generating cylindrical vector beams of arbitrary structure and polarization distribution. By means of a Sagnac interferometer, 


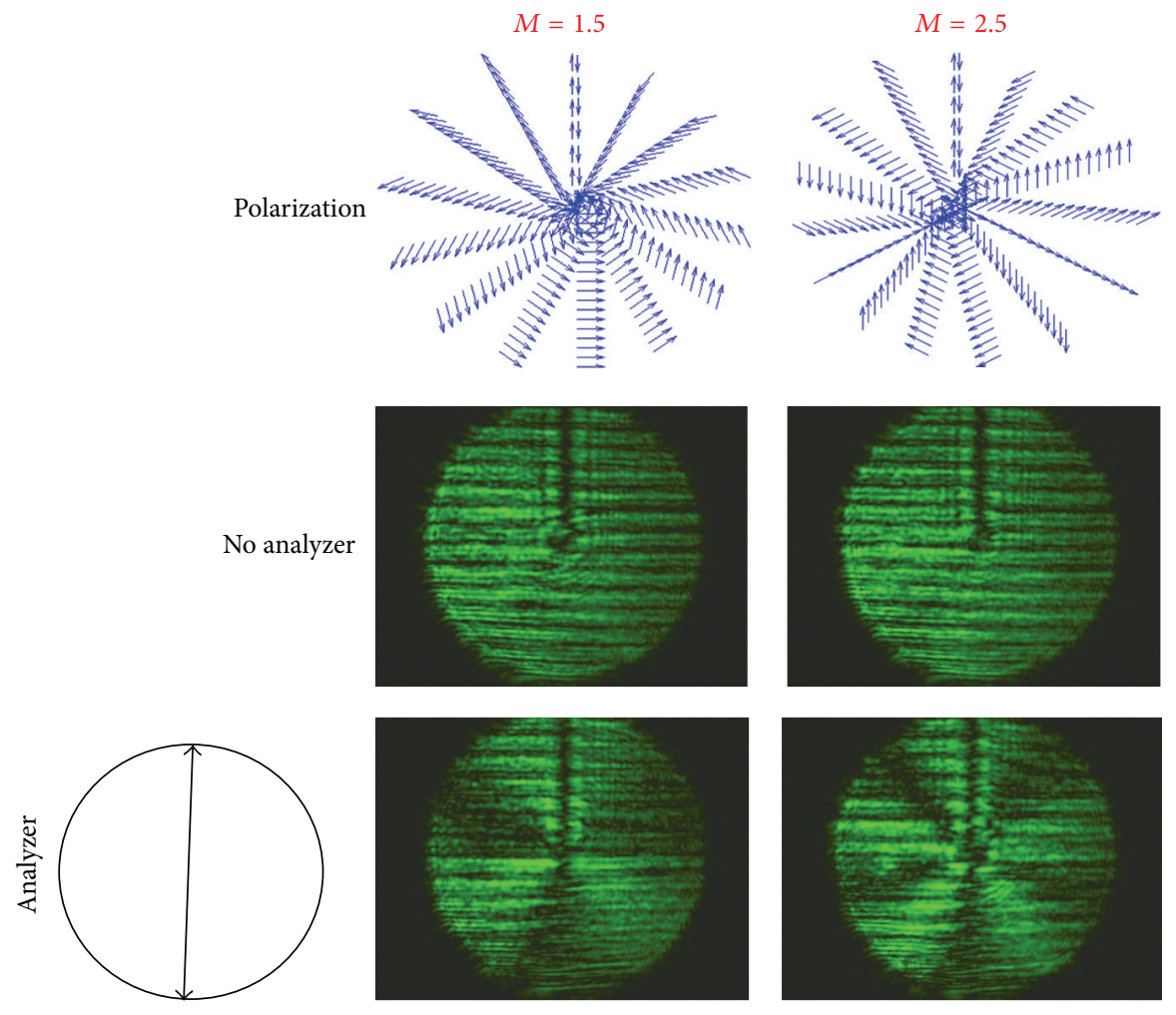

Figure 4: Cylindrical vector beams generated for a noninteger topological charge $M=1.5$ and $M=2.5$ and constant phase difference of $\psi=0$ for both.
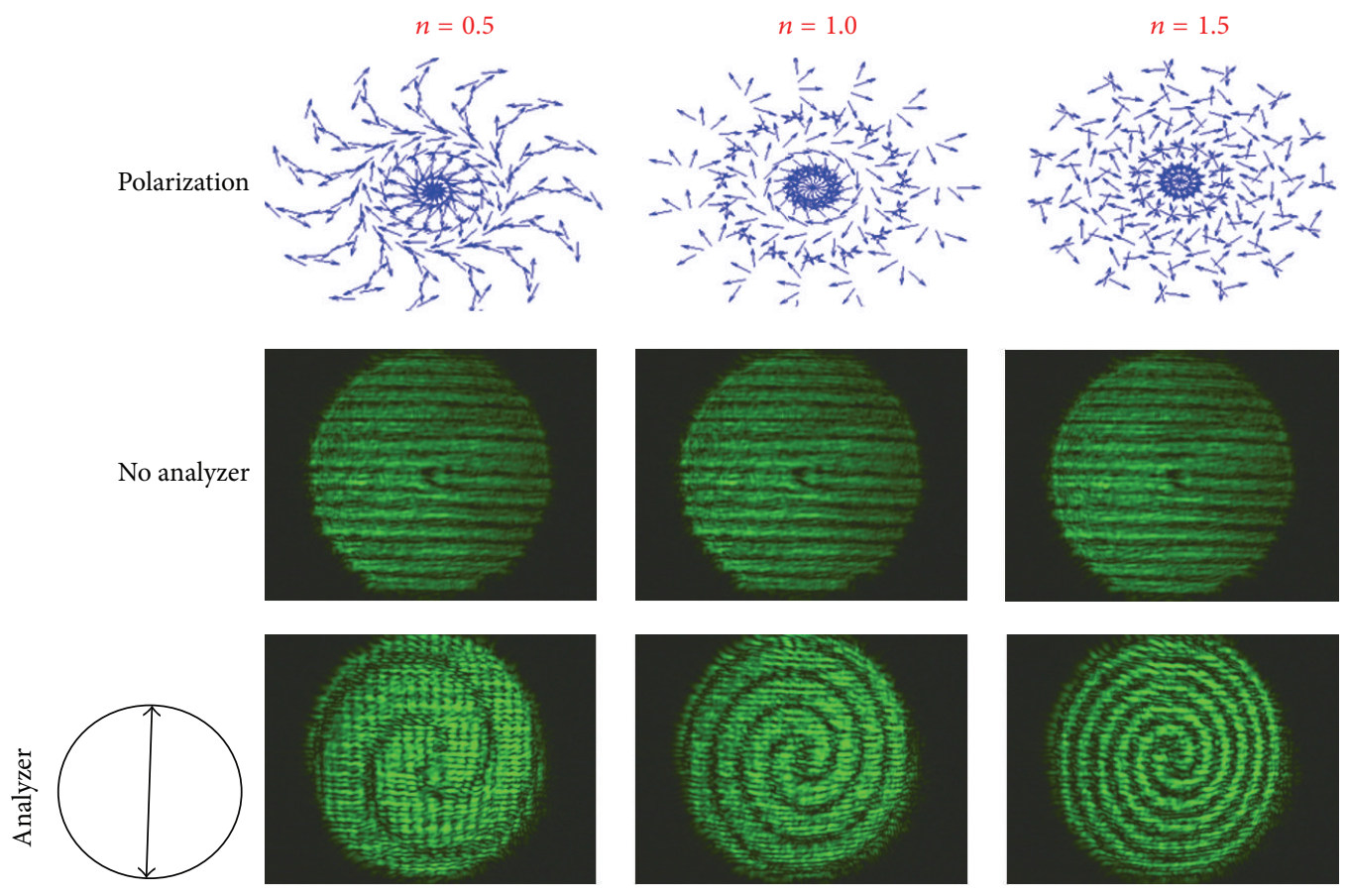

FIGURE 5: Cylindrical vector beams with topological charge $m=1$ and radial numbers $n=0.5,1.0$ and $n=1.5$. 

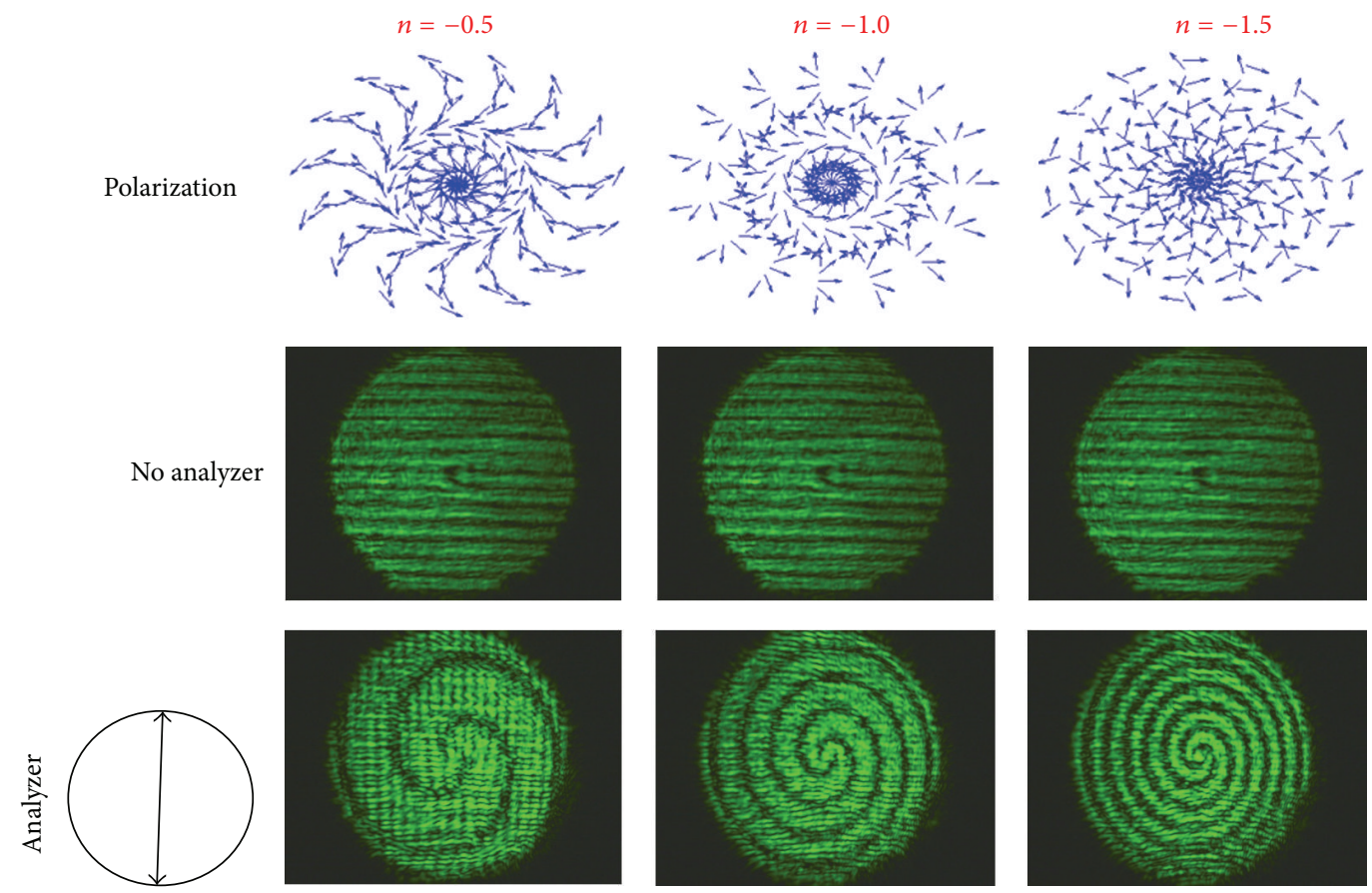

FIGURE 6: Cylindrical vector beams with topological charge $m=1$ and radial numbers $n=-0.5,-1.0$ and $n=-1.5$.

we have demonstrated that any desired polarization can be realized by the appropriate designing CGHs which are projected adjacent to one another, onto a PAL-SLM. The superposition of two orthogonally and coaxially polarized beams is obtained at the output of the Sagnac interferometer. The first diffraction order of the Fourier transform of that output is then analyzed. This experimental setup is stable against environmental disturbances like mechanical vibrations; it is highly reliable and easily reconfigurable. We note however that the energy efficiency of using CGHs to tailor the cylindrical vector beams obtained is relatively low. Nevertheless, in the same experimental geometry, one could substitute the adjacent CGHs by holograms with high diffraction efficiency. This new method provides both a convenient way to investigate the physics of the propagation of cylindrical vector beams and practical means to explore new applications.

\section{Competing Interests}

The authors declare that they have no competing interests.

\section{Acknowledgments}

Support was provided by the Vicepresidencia of Research and Services to Universidad Industrial de Santander (funding Projects 5191/5803 and 5708, both of the institutional program for consolidation of research groups, years 2012 and 2013, resp.). Colciencias was supported through Project 110256934957, "Optics devices for quantum key distribution, high dimensionality systems based in orbital angular momentum of light." Funds were obtained from the National
Call for the Bank of Projects in Science, Technology and Innovation 2012.

\section{References}

[1] D. G. Hall, "Vector-beam solutions of Maxwell's wave equation," Optics Letters, vol. 21, no. 1, pp. 9-11, 1996.

[2] Q. Zhan, "Cylindrical vector beams: from mathematical concepts to applications," Advances in Optics and Photonics, vol. 1, no. 1, pp. 1-57, 2009.

[3] Q. Zhan, "Trapping metallic Rayleigh particles with radial polarization," Optics Express, vol. 12, no. 15, pp. 3377-3382, 2004.

[4] T. A. Nieminen, N. R. Heckenberg, and H. Rubinsztein-Dunlop, "Forces in optical tweezers with radially and azimuthally polarized trapping beams," Optics Letters, vol. 33, no. 2, pp. 122-124, 2008.

[5] M. G. Donato, S. Vasi, R. Sayed et al., "Optical trapping of nanotubes with cylindrical vector beams," Optics Letters, vol. 37, no. 16, pp. 3381-3383, 2012.

[6] D. Naidoo, M. Fromager, K. Ait-Ameur, and A. Forbes, "Radially polarized cylindrical vector beams from a monolithic microchip laser," Optical Engineering, vol. 54, no. 11, article 111304, 2015.

[7] T. J. Gould, J. R. Myers, and J. Bewersdorf, “Total internal reflection STED microscopy," Optics Express, vol. 19, no. 14, pp. 13351-13357, 2011.

[8] Z. Man, C. Min, S. Zhu, and X.-C. Yuan, "Tight focusing of quasi-cylindrically polarized beams," Journal of the Optical Society of America A, vol. 31, no. 2, pp. 373-378, 2014.

[9] P. B. Phua, W. J. Lai, Y. L. Lim et al., "Mimicking optical activity for generating radially polarized light," Optics Letters, vol. 32, no. 4, pp. 376-378, 2007.

[10] G. Machavariani, Y. Lumer, I. Moshe, A. Meir, S. Jackel, and N. Davidson, "Birefringence-induced bifocusing for selection of 
radially or azimuthally polarized laser modes," Applied Optics, vol. 46, no. 16, pp. 3304-3310, 2007.

[11] M. P. Thirugnanasambandam, Y. Senatsky, and K.-I. Ueda, "Generation of radially and azimuthally polarized beams in Yb:YAG laser with intra-cavity lens and birefringent crystal," Optics Express, vol. 19, no. 3, pp. 1905-1914, 2011.

[12] R. Dorn, S. Quabis, and G. Leuchs, "Sharper focus for a radially polarized light beam," Physical Review Letters, vol. 91, no. 23, Article ID 233901, 2003.

[13] Y. Kozawa and S. Sato, "Generation of a radially polarized laser beam by use of a conical Brewster prism," Optics Letters, vol. 30, no. 22, pp. 3063-3065, 2005.

[14] A. Flores-Pérez, J. Hernández-Hernández, R. Jáuregui, and K. Volke-Sepúlveda, "Experimental generation and analysis of first-order TE and TM Bessel modes in free space," Optics Letters, vol. 31, no. 11, pp. 1732-1734, 2006.

[15] K. C. Toussaint Jr., S. Park, J. E. Jureller, and N. F. Scherer, "Generation of optical vector beams with a diffractive optical element interferometer," Optics Letters, vol. 30, no. 21, pp. 2846$2848,2005$.

[16] C. Maurer, A. Jesacher, S. Fürhapter, S. Bernet, and M. RitschMarte, "Tailoring of arbitrary optical vector beams," New Journal of Physics, vol. 9, article 78, 2007.

[17] X.-L. Wang, Y. Li, J. Chen, C.-S. Guo, J. Ding, and H.-T. Wang, "A new type of vector fields with hybrid states of polarization," Optics Express, vol. 18, no. 10, pp. 10786-10795, 2010.

[18] H. Chen, J. Hao, B.-F. Zhang, J. Xu, J. Ding, and H.-T. Wang, "Generation of vector beam with space-variant distribution of both polarization and phase," Optics Letters, vol. 36, no. 16, pp. 3179-3181, 2011.

[19] X.-L. Wang, J. Ding, W.-J. Ni, C.-S. Guo, and H.-T. Wang, "Generation of arbitrary vector beams with a spatial light modulator and a common path interferometric arrangement," Optics Letters, vol. 32, no. 24, pp. 3549-3551, 2007.

[20] V. G. Niziev, R. S. Chang, and A. V. Nesterov, "Generation of inhomogeneously polarized laser beams by use of a Sagnac interferometer," Applied Optics, vol. 45, no. 33, pp. 8393-8399, 2006.

[21] N. Fukuchi, Y. Biqing, Y. Igasaki, N. Yoshida, Y. Kobayashi, and T. Hara, "Oblique-incidence characteristics of a parallel-aligned nematic-liquid-crystal spatial light modulator," Optical Review, vol. 12, no. 5, pp. 372-377, 2005.

[22] J. B. Götte, K. O’holleran, D. Preece et al., "Light beams with fractional orbital angular momentum and their vortex structure," Optics Express, vol. 16, no. 2, pp. 993-1006, 2008.

[23] M. V. Berry, "Optical vortices evolving from helicoidal integer and fractional phase steps," Journal of Optics A: Pure and Applied Optics, vol. 6, no. 2, pp. 259-268, 2004. 

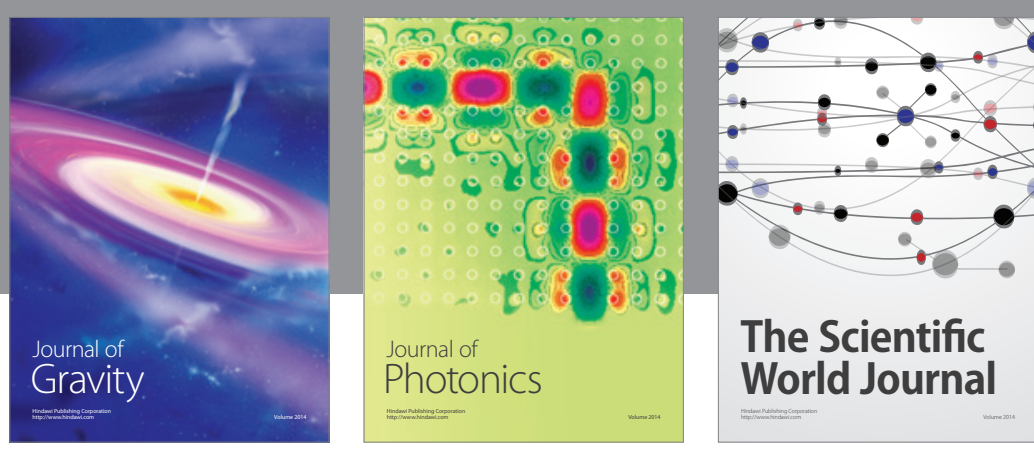

The Scientific World Journal
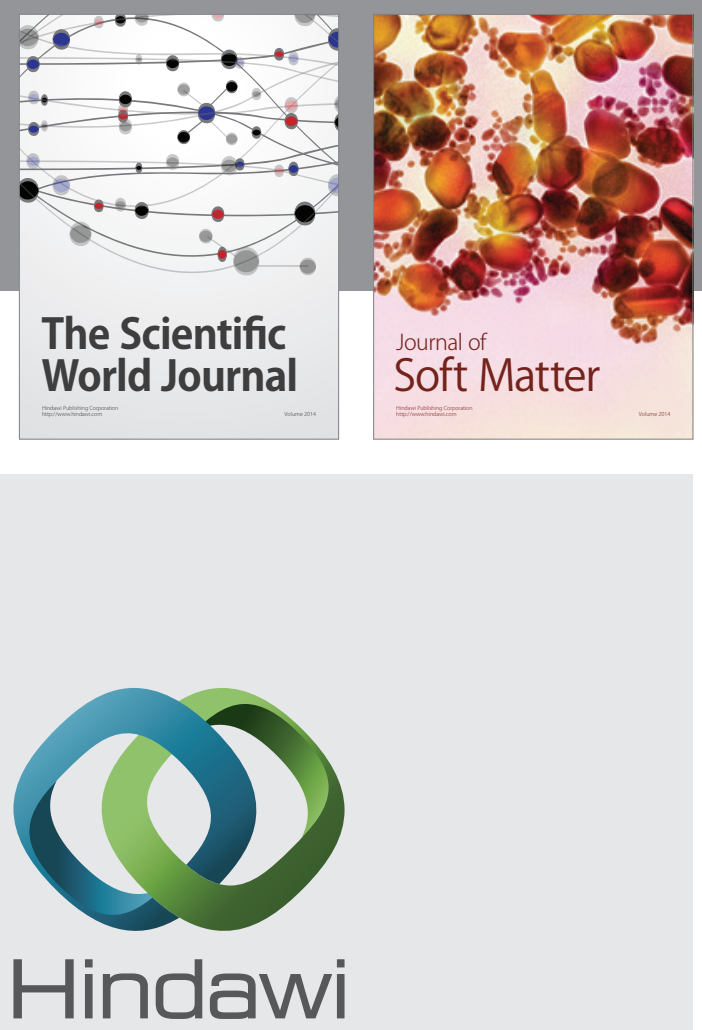

Submit your manuscripts at

http://www.hindawi.com

nternational Journal of

Statistical Mechanics
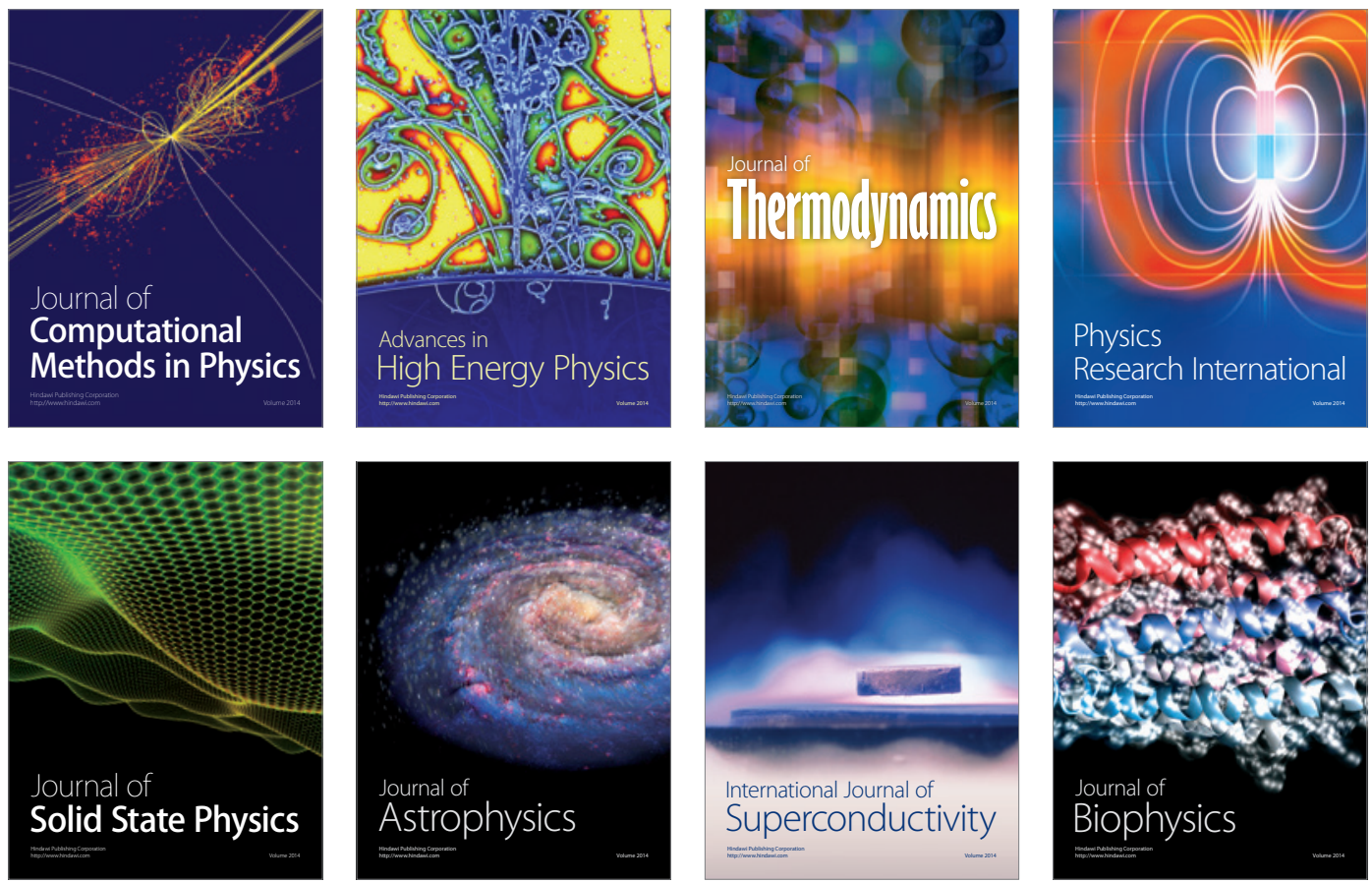
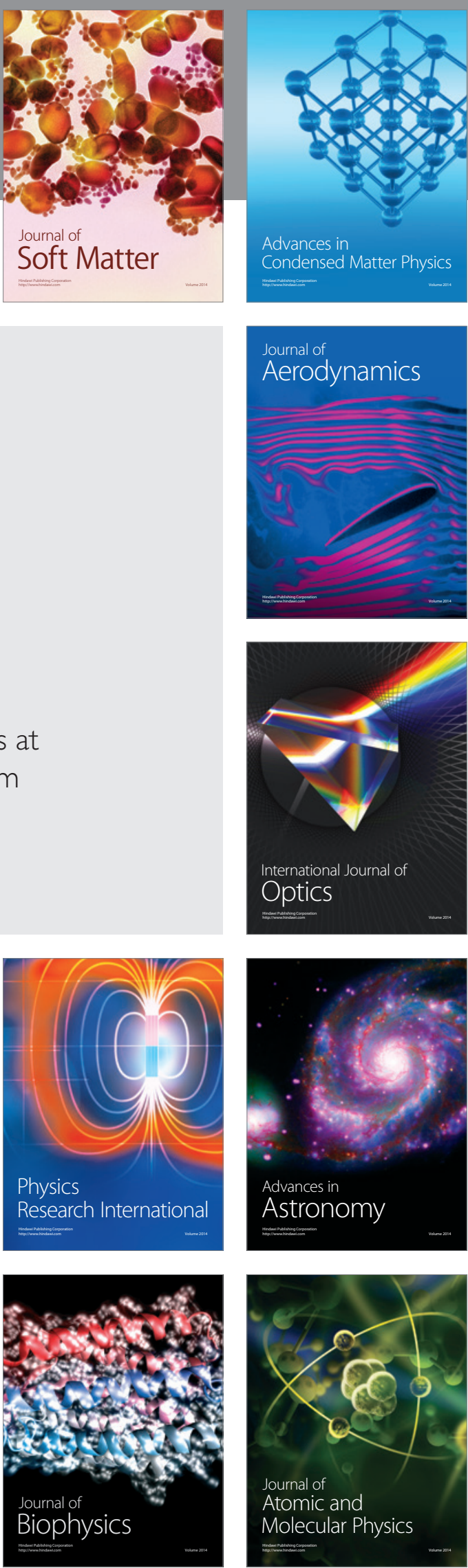